\title{
Relating Franck-Condon blockade to redox chemistry in the single-particle picture
}

Cite as: J. Chem. Phys. 149, 104109 (2018); https://doi.org/10.1063/1.5043480

Submitted: 09 June 2018 . Accepted: 28 August 2018 . Published Online: 14 September 2018

Kirk H. Bevan (D), Antoine Roy-Gobeil (D), Yoichi Miyahara (D), and Peter Grutter
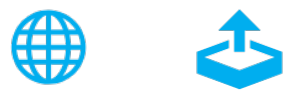

\section{ARTICLES YOU MAY BE INTERESTED IN}

Perspective: Theory of quantum transport in molecular junctions

The Journal of Chemical Physics 148, 030901 (2018); https://doi.org/10.1063/1.5003306

Electron transfer from the perspective of electron transmission: Biased non-adiabatic intermolecular reactions in the single-particle picture

The Journal of Chemical Physics 146, 134106 (2017); https://doi.org/10.1063/1.4979572

Beyond Marcus theory and the Landauer-Büttiker approach in molecular junctions: A unified framework

The Journal of Chemical Physics 149, 154112 (2018); https://doi.org/10.1063/1.5049537
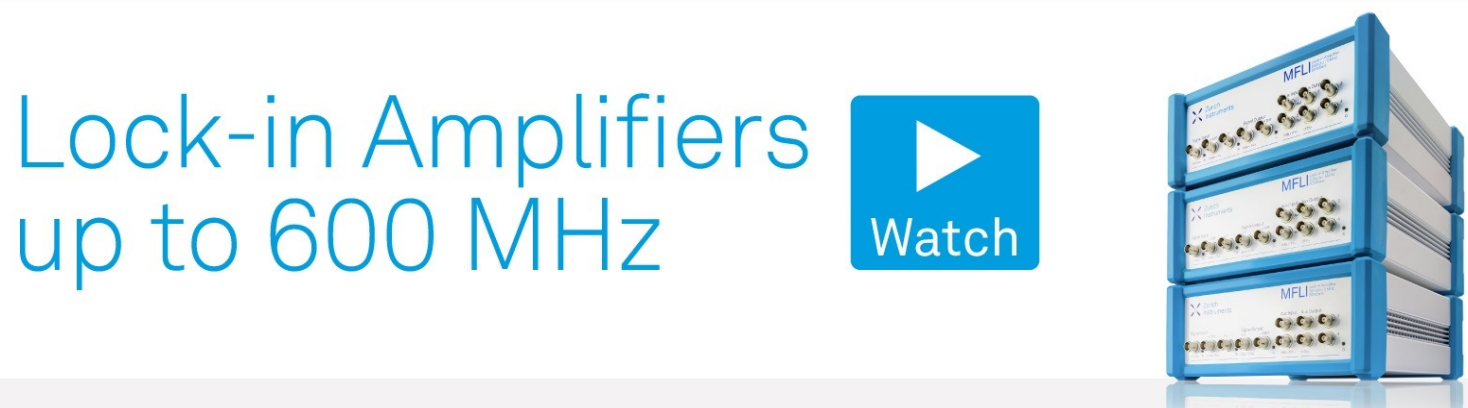

J. Chem. Phys. 149, 104109 (2018); https://doi.org/10.1063/1.5043480 


\title{
Relating Franck-Condon blockade to redox chemistry in the single-particle picture
}

\author{
Kirk H. Bevan, ${ }^{1, a)}$ Antoine Roy-Gobeil, ${ }^{2}$ Yoichi Miyahara, ${ }^{2}$ and Peter Grutter ${ }^{2}$ \\ ${ }^{1}$ Division of Materials Engineering, Faculty of Engineering, McGill University, Montréal, \\ Québec H3A OC5, Canada \\ ${ }^{2}$ Department of Physics, McGill University, 3600 Rue University, Montréal, Québec H3A 2T8, Canada
}

(Received 9 June 2018; accepted 28 August 2018; published online 14 September 2018)

\begin{abstract}
In this work, we explore Franck-Condon blockade in the "redox limit," where nuclear relaxation processes occur much faster than the rate of electron transfer. To this end, the quantized rate expressions for electron transfer are recast in terms of a quantized redox density of states (DOS) within a single phonon mode model. In the high temperature regime, this single-particle picture formulation of electron transfer is shown to agree well with the semi-classical rate and DOS expressions developed by Gerischer and Hopfield. Upon incorporation into a two electrode formulation, utilizing the master equation approach, the low temperature quantized conductance features of Franck-Condon blockade are reproduced. Moreover, at sufficiently large reorganization energies, it is argued that Franck-Condon blockade should also be observable in room temperature systems. In general, this work aims to further bridge descriptions of electron transfer and transport in the single-particle picture. Published by AIP Publishing. https://doi.org/10.1063/1.5043480
\end{abstract}

\section{INTRODUCTION}

Two electrode systems exhibit a fascinating range of dimension dependent conduction phenomena. ${ }^{1-3}$ For example, with decreasing dimensionality, there is often a corresponding increase in electron-electron and electron-phonon interactions. ${ }^{2-5}$ In systems where both probes are weakly coupled to an intermediary species (e.g., the tunneling regime), the increased interaction between electrons at small dimensions gives rise to a charging cost $U$ for the addition/removal of each electron and results in Coulomb blockade [as illustrated in Fig. 1(a)]. This first becomes manifest at mesoscopic length scales, with metallic nanoparticles forming an archetypal platform. ${ }^{2,3,6-8}$ As the dimensionality of the intermediary species is decreased further to the molecular regime, the charging energy $U$ continues to rise and electronphonon coupling becomes more prominent leading to the subsequent regime of Franck-Condon blockade [illustrated in Fig. 1(b)]. ${ }^{4-9-11}$ Franck-Condon blockade is characterized by the suppression of current flow at small applied biases $\left(V_{B}\right)$, when a Coulomb blockaded single-particle level would otherwise produce high conductance. ${ }^{4,9-11}$ Fundamentally, it arises due to nuclear reorganization, a manifestation of electron-phonon coupling, which occurs upon transitioning between charge states in the molecular size regime., ${ }^{4,9}$ This manifests itself as an enhancement of the off-diagonal Franck-Condon coupling factors ${ }^{12,13}$ and corresponding suppression in diagonal coupling factors, between the nuclear wavefunctions of the intermediary species [such as the molecule shown in Fig. 1(b)] as it transitions between charge states. $^{4,9}$

a)Electronic mail: kirk.bevan@mcgill.ca
Yet, as we show in this work, this mathematically formal perspective on Franck-Condon blockade can be intuitively explored by adopting and integrating some of the concepts developed within the single-particle approach to electron transfer theory. ${ }^{14-18}$ Specifically, in the "redox limit" where a molecular species reorganizes/relaxes its nuclear coordinates much faster than the rate of electron tunneling to/from the contacts, Franck-Condon blockade may also be viewed as the manifestation of successive electron transfer (tunneling) redox reactions. ${ }^{11,19-22}$ Consider the scenario where an electron tunnels first from the left contact to an oxidized molecule with $N-1$ electrons [see Fig. 1(b)]. Subsequently, after nuclear relaxation/reorganization, the reduced molecule with $N$ electrons then gives up its electron to the right contact. In this manner, two successive redox reactions have mediated electron transport resulting in Franck-Condon blockade. ${ }^{11,19-22}$ Important work on the intersection between electron transport and electron transfer (redox chemistry) has been made by the Levich-Dogonadze school and others. ${ }^{4,9,19-21,23-27}$

This redox based perspective on Franck-Condon blockade is appealing because it naturally lends itself to the semiclassical single-particle electron transfer formalisms developed by Hopfield and Gerischer. ${ }^{14-17}$ The single-particle picture is the standard approach for describing both the Coulomb and Franck-Condon blockade phenomena (as well as electron transport and molecular conduction phenomena, in general). ${ }^{2-4,9,28,29}$ However, the Gerischer and Hopfield formalisms were developed to describe high temperature redox processes (e.g., room temperature) and Franck-Condon blockade has been primarily observed only at very low temperatures (e.g., $\sim 4 \mathrm{~K}$ ). ${ }^{10,11}$ This then, leads to two primary questions which we focus upon in this work. First, how does one formally describe Franck-Condon blockade at low temperatures through a redox oriented single-particle 
(a) Mesoscopic

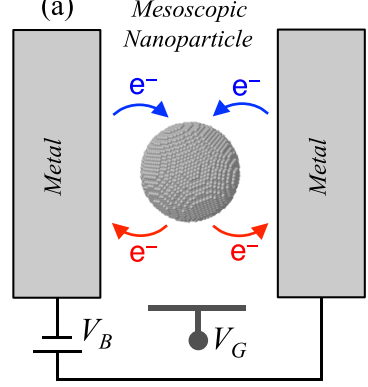

(b)

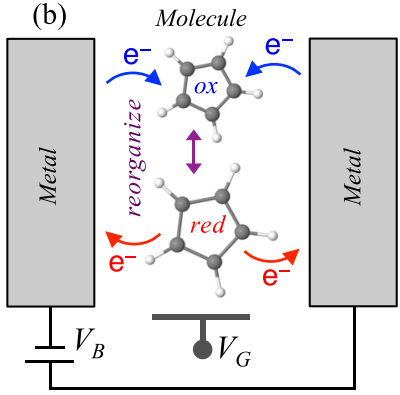

FIG. 1. (a) A mesoscopic nanoparticle undergoing Coulomb blockade. (b) A molecule undergoing Franck-Condon blockade in the "redox limit" (an extreme form of Coulomb blockade). In either case, the species mediates electron flow between two contacts subject to a bias $V_{B}$. A further gate potential $V_{G}$ is applied to shift the single-particle states of the species and vary the two-electrode conduction properties. In the configuration envisioned, electrons are transferred via tunneling to/from each contact. Chemically, when a species gains an electron, it transforms from an oxidized $(o x)$ state to a reduced (red) state and vice versa.

perspective? ${ }^{11,14-17,22}$ Second, is there any reason why Franck-Condon blockade might not also be observed at high temperatures, given the prevalence of room temperature redox phenomena? $?^{2,30}$ To address the first question, we begin this work by recasting models of quantized electron transfer in terms of a redox density of states (DOS), ${ }^{14-18,31,32}$ taking into account the strengthening of off-diagonal FranckCondon coupling factors with increased electron-phonon coupling (Sec. II). ${ }^{4,12,13}$ Our approach is restricted to first-order perturbation and hence to non-adiabatic electron transfer. This model is shown to produce electron transfer rate quantization at low temperatures and excellent agreement with the semi-classical Gerisher-Hopfield ${ }^{14-17}$ rate models in the high temperature limit. Subsequently, we utilize this rate quantization model to produce the characteristic features of the low temperature Franck-Condon blockade by employing a master equation approach (Sec. III). ${ }^{2,4,9-11}$ From this low temperature Franck-Condon based redox perspective, we are able to extrapolate further, utilizing comparable reorganization energies and rates observed in the literature measured for room temperature redox phenomena, ${ }^{30,32}$ to argue that Franck-Condon blockade should also be observable in the high temperature regime-via the drastic suppression of low bias current flow. ${ }^{19-21}$

\section{ELECTRON TRANSFER RATE MODELS}

\section{A. Mesoscopic regime}

Let us begin with the picture in Fig. 2(a), depicting electron transfer (tunneling) between a mesoscopic nanoparticle and a metal interface. The single-particle energy $(\varepsilon)$ at which an electron may be added to such a nanoparticle is given by $\varepsilon_{d}=E(N)-E(N-1)$ as shown in Fig. 2(b), where $E(N)$ is the nanoparticle's total energy with $N$-electrons. Now, there is a charging energy $U$ cost related to the particle's dimensionality that simplifies to $U=q^{2} / 4 \pi \epsilon R$ for a spherical particle with radius $R$-where, $q$ is the charge of an electron and $\epsilon$ is the electric permittivity. ${ }^{2}$ When $U \gg k_{B} T$, the phenomena (a)
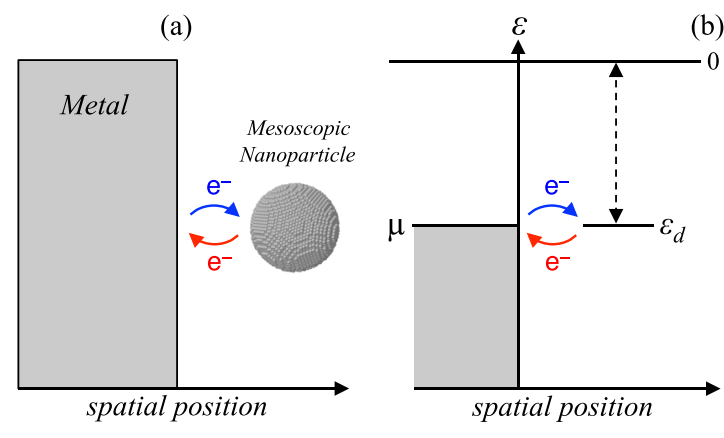

(b) $E$

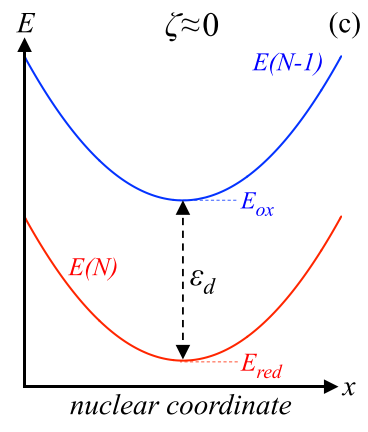

(d)
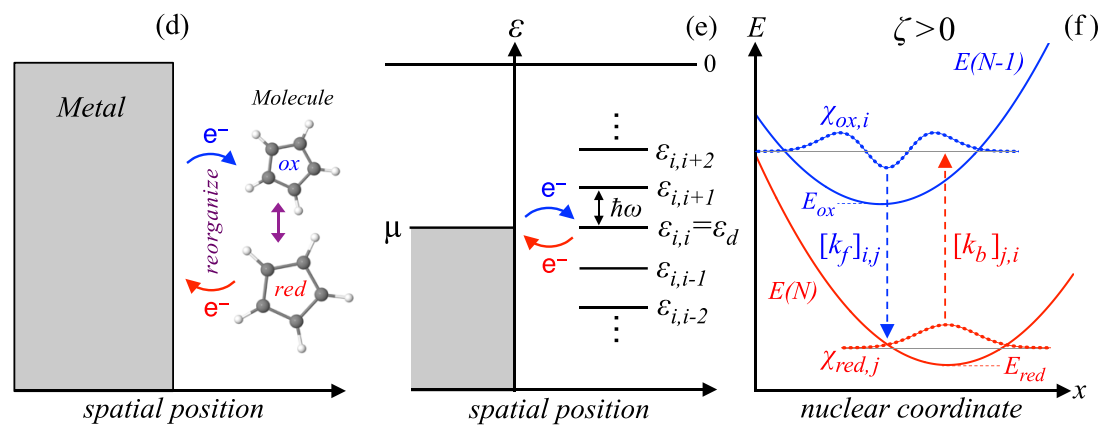

FIG. 2. (a) Schematic of electron transfer to a mesoscopic particle with a charging energy $(U)$. (b) Single-particle picture of electron transfer to a mesoscopic particle at an energy level $\varepsilon_{d}$. (c) The corresponding total energy description of a mesoscopic nanoparticle with near zero electron-phonon coupling $(\zeta \approx 0)$. The minimum total energy for the $N-1$ electron oxidized state is $E_{o x}$, and that for the $N$ electron reduced state is $E_{r e d}$. (d) Schematic of electron transfer to a molecule, with nuclear coordinate reorganization. (e) Single-particle picture of molecular electron transfer to $\varepsilon_{d}$ and also to levels situated at intervals of $\hbar \omega$, with the propensity determined by Franck-Condon coupling. (f) Total energy picture of electron transfer to a molecule, with a corresponding shift in the nuclear coordinate minimum to sizable electron-phonon coupling $(\zeta>0)$-or sizable reorganization, in the chemistry terminology. The nuclear wavefunctions in the oxidized and reduced states are denoted by $\chi_{o x}$ and $\chi_{\text {red }}$, respectively. Forward and backward electron transfer rates $\left(k_{f}\right.$ and $\left.k_{b}\right)$ between nuclear configurations are determined by the Franck-Condon coupling factors. 
of Coulomb blockade becomes manifest, whereby a further energy of $U$ must be input (typically through an external gate) to access the next charge state with $N+1$ electrons (an so on for adding/removing further electrons). For a detailed discussion on Coulomb blockade see, for example, Refs. 2 and 3 .

The mesoscopic regime is typically characterized by relatively weak coupling between electrons and nuclei. This is due to the dimensionality of the nanoparticle, whereby electrons remain relatively delocalized (assuming a metallic nanoparticle) such that the nuclear coordinates $(x)$ are relatively unperturbed by the addition/removal of an electron. In this work, we characterize electron-phonon coupling by the dimensionless parameter $\zeta{ }^{33-35}$ In the mesoscopic regime, $\zeta \approx 0 .{ }^{4,9}$ This physics is displayed in Fig. 2(c), whereby the total energies $E(N-1)$ and $E(N)$ [for $N-1$ and $N$ electrons] retain the same nuclear coordinate minima. This means, that regardless of the internal nuclear coordinate $x$ of a mesoscopic nanoparticle, the single-particle energy will be $\varepsilon_{d}$ (for adding/removing the $N$ th electron) due to the orthogonality of nuclear wavefunctions (when $\zeta \approx 0$ ). Thus in the mesoscopic regime, the forward electron transfer rate to a nanoparticle (with $N-1$ electrons) from a metallic contact is approximated by $18,31,36$

$$
\lim _{\zeta \rightarrow 0} k_{f}=\frac{2 \pi}{\hbar} \int f(\varepsilon)|M|^{2} D_{S} \delta\left(\varepsilon-\varepsilon_{d}\right) d \varepsilon .
$$

Likewise, the backward electron transfer rate from a nanoparticle (with $N$ electrons) in the mesoscopic regime can be expressed as

$$
\lim _{\zeta \rightarrow 0} k_{b}=\frac{2 \pi}{\hbar} \int[1-f(\varepsilon)]|M|^{2} D_{S} \delta\left(\varepsilon-\varepsilon_{d}\right) d \varepsilon .
$$

Here $|M|^{2}$ is the electronic coupling between the metal and nanoparticle, evaluated in terms of single-particle wavefunctions, $D_{S}$ is the density-of-states (DOS) inside the contact/metal, and $\hbar$ is Planck's constant. Usually, $|M|^{2}$ and $D_{S}$ are approximated as constants that are brought out of the single-particle energy integral. ${ }^{31,37}$ The Fermi function $f(\varepsilon)=1 /\left[1+\exp \left((\varepsilon-\mu) / k_{B} T\right)\right]$ delineates filled and occupied states inside the contact $-\mu$ is its electrochemical potential, $k_{B}$ is Boltzmann's constant, and $T$ is the system temperature. Finally, it is important to note that Eqs. (1) and (2) are only valid in the regime where the separation between energy levels is much larger than $k_{B} T$. However, in this work, we will only consider one level at all temperatures to simplify the comparison with Franck-Condon blockade phenomena.

\section{B. Molecular regime}

\section{Formalism in the molecular limit}

On the other hand, the molecular size regime is typically characterized by strong electron-phonon coupling. ${ }^{4,5,9-11,22}$ Meaning, there is sizable reorganization of nuclear coordinates when a molecule transitions between $N-1$ and $N$ electrons as shown in Fig. 2(d). Thus, the molecular regime is often characterized by $\zeta>0$. Coulomb blockade is still present in a molecular system, albeit in an extreme form, since the charging energy only increases with reduced dimensionality. ${ }^{3,6,28,38}$ However, the presence of strong electron-phonon coupling opens up new probable single-particle electron transfer energies about $\varepsilon_{d}$ as shown in Fig. 2(e).,5 These additional single-particle energies are separated by $\hbar \omega$, in terms of the molecular phonon frequency $(\omega)$. Here we only consider one phonon mode, to simplify the discussion, but the argument may be extended to multiple phonon modes. ${ }^{4,39}$ During the remainder of this discussion, we will seek to understand these "nuclear reorganization assisted" transitions in the framework of both Coulomb blockade and redox chemistry in the single-particle picture.

In each charge state, the total energy $(E)$ of the molecule may be approximated as a harmonic oscillator,

$$
\begin{aligned}
E(N) & =E_{r e d}+\frac{1}{2} m \omega^{2} x^{2}, \\
E(N-1) & =E_{o x}+\frac{1}{2} m \omega^{2}(x+\Delta x),{ }^{2}
\end{aligned}
$$

where $m$ is the oscillator mass. As shown in Fig. 2(f), $E_{\text {red }}$ is the total energy at the nuclear coordinate minimum in the $N$ electron (reduced) state and $E_{o x}$ is the total energy at the nuclear coordinate minimum in the $N-1$ electron (oxidized) state. The shift in nuclear coordinates, which occurs due to electron-phonon coupling, is given by $\Delta x=\zeta \sqrt{2 \hbar / m \omega}$. 4 The accessible phonon energies in the oxidized and reduced state are given by $E_{o x, i}=E_{o x}+\left(i+\frac{1}{2}\right) \hbar \omega$ and $E_{r e d, j}=E_{\text {red }}$ $+\left(j+\frac{1}{2}\right) \hbar \omega$, respectively. These phonon energies have corresponding nuclear wavefunctions $\chi_{o x, i}$ and $\chi_{\text {red }, j}$ as shown in Fig. 2(f), for the reduced $(N)$ and oxidized $(N-1)$ charge states, respectively.

Now, during an electron transfer event from the contact to the molecule, the molecule must transition between nuclear configurations from $\chi_{o x, i}$ to $\chi_{\text {red }, j}$ [see Fig. 2(f)]. Likewise, when an electron is transferred from the molecule to the contact, the molecule transitions from $\chi_{\text {red }, j}$ to $\chi_{o x, i}$ [see Fig. 2(f)]. The propensity of a given nuclear transition is dictated by the Franck-Condon coupling factors $\left|\left\langle\chi_{o x, i}\right\rangle \chi_{\text {red }, j}\right|^{2}$ and $\left|\left\langle\chi_{\text {red }, j}\right\rangle \chi_{o x, i}\right|^{2}$, for the forward and backward electron transfer rates, respectively. These transitions correspond to single-particle energies $\varepsilon_{i, j}=\varepsilon_{d}+(j-i) \hbar \omega$, that are situated about the single-particle energy $\varepsilon_{d}=E_{\text {red }}-E_{o x}$ in intervals of $\hbar \omega$ as depicted in Fig. 2(e) and discussed earlier. The Franck-Condon couplings weigh upon the rate of electron transfer to each single-particle energy $\left(\varepsilon_{i, j}\right)$, resulting in forward and backward rate equations of the form

$$
\begin{aligned}
& {\left[k_{f}\right]_{i, j}=\frac{2 \pi|M|^{2} D_{S}}{\hbar} \int f(\varepsilon)\left|\left\langle\chi_{o x, i}\right\rangle \chi_{r e d, j}\right|^{2} \delta_{i, j}(\varepsilon) d \varepsilon} \\
& {\left[k_{b}\right]_{j, i}=\frac{2 \pi|M|^{2} D_{S}}{\hbar} \int[1-f(\varepsilon)]\left|\left\langle\chi_{r e d, j}\right\rangle \chi_{o x, i}\right|^{2} \delta_{i, j}(\varepsilon) d \varepsilon}
\end{aligned}
$$

for each possible transition between nuclear configurations upon adding or removing an electron-where, $\delta_{i, j}(\varepsilon)=\delta(\varepsilon$ $\left.-\varepsilon_{i, j}\right)$. The total forward $\left(k_{f}\right)$ and backward $\left(k_{b}\right)$ rates, in the molecular limit, are a summation across all possible transitions weighted by the probability $\left(P_{i}\right)$ of residing in an initial nuclear configuration 


$$
\begin{aligned}
k_{f} & =\sum_{j} \sum_{i}\left[k_{f}\right]_{i, j} P_{i} \\
& =\frac{2 \pi|M|^{2} D_{S}}{\hbar} \int f(\varepsilon) D_{o x}(\varepsilon) d \varepsilon, \\
k_{b} & =\sum_{i} \sum_{j}\left[k_{b}\right]_{j, i} P_{j} \\
& =\frac{2 \pi|M|^{2} D_{S}}{\hbar} \int[1-f(\varepsilon)] D_{\text {red }}(\varepsilon) d \varepsilon,
\end{aligned}
$$

where $D_{o x}(\varepsilon)$ and $D_{\text {red }}(\varepsilon)$ are the oxidized and reduced DOS distributions of the molecule, respectively (in the spirit of Gerischer and Hopfield). ${ }^{14-17}$ In the limit $\zeta \rightarrow 0$, Eqs. (7) and (8) reduce to the more familiar tunneling expressions in Eqs. (1) and (2).

The redox DOS expressions in Eqs. (7) and (8) may be written in the quantum regime as 34,35

$$
\begin{aligned}
& D_{o x}=\sum_{j} \sum_{i} P_{i}\left|\left\langle\chi_{o x, i}\right\rangle \chi_{r e d, j}\right|^{2} \delta\left(\varepsilon-\varepsilon_{i, j}\right), \\
& D_{\text {red }}=\sum_{i} \sum_{j} P_{j}\left|\left\langle\chi_{r e d, j}\right\rangle \chi_{o x, i}\right|^{2} \delta\left(\varepsilon-\varepsilon_{i, j}\right) .
\end{aligned}
$$

For the parabolic modes assumed here, the Franck-Condon overlap integrals can be solved analytically as described in Ref. 4. Moreover, the occupation probability of a given nuclear configuration is dictated by the partition function, which takes the below form if we assume a Boltzmann distribution for the occupation of phonon modes

$$
P_{i} \approx e^{-i \hbar \omega / k_{B} T}\left[1-\exp \left(-\hbar \omega / k_{B} T\right)\right]
$$

for the $i$ th phonon state in either the reduced or oxidized configuration. The impact upon the redox DOS, and rate behavior, when a Bose-Einstein distribution is utilized shall be explored in later work. In the semi-classical limit, when $\hbar \omega$ is sufficiently smaller than $k_{B} T$, the redox DOS distributions take on the well-known Gerischer-Hopfield semi-classical form

$$
\begin{aligned}
& D_{o x, c l}=\frac{1}{\sqrt{4 \pi \lambda k_{B} T}} \exp \left(\frac{-\left(\varepsilon-\varepsilon_{o x}\right)^{2}}{4 \lambda k_{B} T}\right), \\
& D_{r e d, c l}=\frac{1}{\sqrt{4 \pi \lambda k_{B} T}} \exp \left(\frac{-\left(\varepsilon-\varepsilon_{r e d}\right)^{2}}{4 \lambda k_{B} T}\right)
\end{aligned}
$$

assuming a Boltzmann distribution in the same manner as Gerischer. $^{14-18,34,35}$ Here $\lambda=\zeta^{2} \hbar \omega$ is the heterogeneous reorganization energy, which is related to the oxidation and reduction single-particle energies through $\varepsilon_{o x}=\varepsilon_{d}+\lambda$ and $\varepsilon_{\text {red }}=\varepsilon_{d}-\lambda .^{4,18,31}$ Finally, it is important to note that this "redox" rate picture ignores co-tunneling processes, under the assumption that electronic coupling $\left(|M|^{2}\right)$ is very weak. ${ }^{4,40}$ An analysis exploring the formal relationship between this redox based rate picture and more general descriptions of FranckCondon blockade, particularly those provided in Ref. 9, can be found in the supplementary material.

\section{Analysis of molecular limit rates and distributions}

In Fig. 3, we have plotted the redox DOS distributions and corresponding rates at two reorganization energies. For the remainder of this discussion, we will primarily characterize systems by the magnitude of their reorganization energy
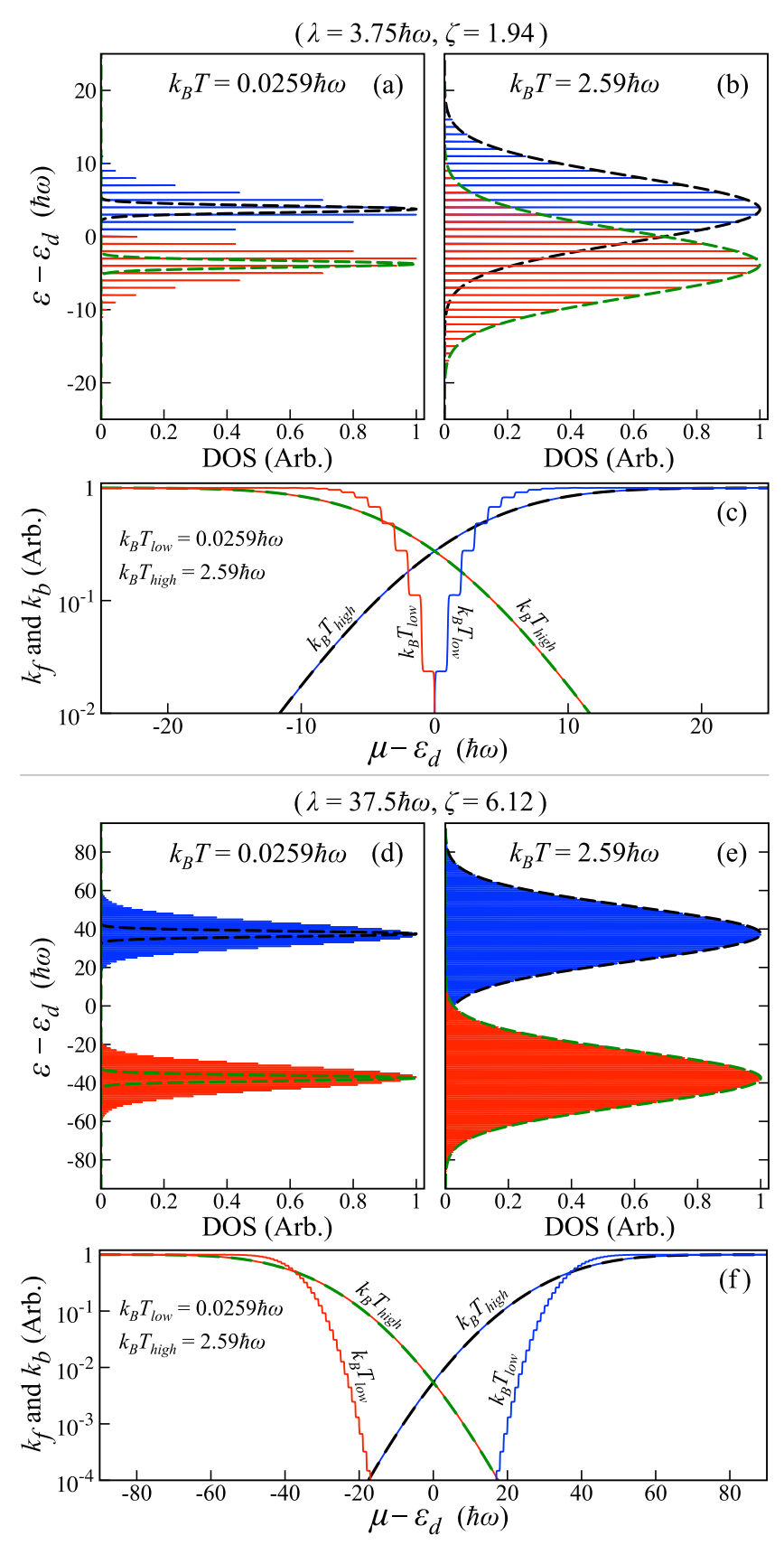

FIG. 3. Quantized redox DOS distributions for reduced (red) and oxidized (blue) states at: (a) $k_{B} T=0.0259 \hbar \omega$ and $\zeta=1.94$; (b) $k_{B} T=2.59 \hbar \omega$ and $\zeta=1.94$; (d) $k_{B} T=0.0259 \hbar \omega$ and $\zeta=6.12$; and (e) $k_{B} T=2.59 \hbar \omega$ and $\zeta=6.12$. The corresponding semi-classical redox DOS and rates are shown in green and black, for the reduced and oxidized states, respectively. Forward and backward $\left(k_{f}\right.$ and $k_{b}$ ) electron transfer rates are shown in red and blue, respectively, at high and low temperatures for: (c) $\zeta=1.94$ and (f) $\zeta=$ 6.12. Again, the semi-classical redox rates are shown in green and black at $k_{B} T=2.59 \hbar \omega$, for the reduced and oxidized states, respectively. The reorganization energy is related to $\zeta$ via $\lambda=\zeta^{2} \hbar \omega$.

$\left(\lambda=\zeta^{2} \hbar \omega\right)$, as this provides a proportional means of interpreting spectra measured in the single-particle picture-rather than quadratic, as is the case for $\zeta .^{18,31,32,37,38}$ Moreover, we will also simply refer to $\lambda$ as the "reorganization energy" (rather than the "heterogeneous reorganization energy"). ${ }^{18,31,37}$

Let us begin by considering a relatively small reorganization energy at $\lambda=3.75 \hbar \omega$ (corresponding to $\zeta=1.94$ ) in the low temperature regime as shown in Fig. 3(a). Here it can be 
seen that the quantum form of the redox DOS given by Eqs. (9) and (10), shown in red and blue, respectively, results in a highly discretized distribution at $k_{B} T=0.0259 \hbar \omega$. Plotted atop these quantum distributions, we have juxtaposed the results for the semi-classical results given by Eqs. (12) and (13), represented in dashed black and blue, respectively. From Fig. 3(a), it can be seen that the quantum distribution differs markedly from the classical distribution at low temperatures, both in breadth and overall shape. This is because, at sufficiently low temperatures (e.g., $k_{B} T=0.0259 \hbar \omega$ ) only the lowest lying phonon mode is occupied during thermal equilibrium $\left[P_{0} \approx 1\right.$ in Eq. (11)]. Thus the low temperature redox DOS distributions are dominated by the Franck-Condon coupling constants and do not necessarily follow a semi-classical Gaussian distribution. This means that there is a fundamental minimum spread in the breadth of redox DOS distributions, that is present irrespective of the temperature (even at $T=0$ ), that is dependent on $\zeta$. Consequently, the semi-classical description is not applicable at very low temperatures.

However, as shown in Fig. 3(b), upon raising the thermal energy sufficiently (e.g., to $k_{B} T=2.59 \hbar \omega$ ), the semi-classical description becomes a fair approximation. Though the quantum distributions remain discrete, at intervals of $\hbar \omega$, they follow the classical redox redistributions quite closely - we are still utilizing $\lambda=3.75 \hbar \omega$. This occurs because further phonon modes are populated at higher temperatures, in the equilibrium configuration of either the $N$ or $N-1$ charge state. This enables a wider range of occupation weighted Franck-Condon overlap terms that produce physics well-described by the familiar Gerischer-Hopfield type distribution. ${ }^{14-17}$

Experimentally, one measures electron transfer rates, which are essentially integral functions of $D_{o x}$ and $D_{\text {red }}$ convolved with the contact Fermi distribution as given by Eqs. (7) and (8). In Fig. 3(c), we have plotted $k_{f}$ and $k_{b}$ in these same high and low temperature regimes $\left(k_{B} T=0.0259 \hbar \omega\right.$ and $\left.k_{B} T=2.59 \hbar \omega\right)$. At $k_{B} T=0.0259 \hbar \omega$, we can clearly see that the quantum redox distributions given by Eqs. (9) and (10) result in quantized steps of $k_{f}$ and $k_{b}$ (shown in blue and red, respectively) as the Fermi level $(\mu)$ of the contact is varied. However, when the temperature is raised sufficiently, the quantized high temperature distributions [given by Eqs. (9) and (10) and displayed in Fig. 3(b)] no longer provide discrete steps in $k_{f}$ and $k_{b}$ [see the red and blue high temperature curves in Fig. 3(c)]. This high temperature smoothening of $k_{f}$ and $k_{b}$ occurs because the Fermi distribution in the contact is also smeared out at higher temperatures and thereby provides a further smoothening convolution effect on the overall rate integral. In fact, the semi-classical and quantum rates agree very well at high temperatures. This can be seen by comparing the semi-classical dashed-black and quantum blue high temperature values of $k_{f}$ in Fig. 3(c), as well as the semi-classical dashed-green and quantum red high temperature values of $k_{b}$.

Similar trends are shown for a larger reorganization energy value of $\lambda=37.5 \hbar \omega$ (corresponding to $\zeta=6.12$ ) in Figs. 3(d)3(f). Again, at temperatures well below $\hbar \omega$, a minimum breadth in the redox DOS is maintained by the Franck-Condon coupling factors (determined by $\zeta$ ). This is evident through the strong disagreement between the semi-classical (dashed black and green) and quantum redox DOS distributions (red and blue) in Fig. 3(d). Since a larger reorganization energy is present in this second example, the discrete nature of the quantum redox DOS is less prominent at both high and low temperatures [see Figs. 3(d) and 3(e)]. Importantly, this leads to an even more continuous agreement between the semi-classical and the quantum redox distributions at high temperatures [see the $k_{B} T=2.59 \hbar \omega$ results in Fig. 3(e)]. However, the discrete nature of the redox distribution at low temperatures $\left(k_{B} T\right.$ $=0.0259 \hbar \omega$ ) can be more clearly seen in the corresponding $k_{f}$ and $k_{b}$ plots in Fig. 3(f) (drawn in blue and red, respectively). But again, convolution of the broadened Fermi distribution in the rate integrals at higher temperatures $\left(k_{B} T=2.59 \hbar \omega\right)$ leads to smooth trends in the quantum rates which compare well with the semi-classical result. To see this, compare the blue $\left(k_{f}\right)$ and red $\left(k_{b}\right)$ quantum curves with the black $\left(k_{f}\right)$ and green $\left(k_{f}\right)$ semi-classical results at $k_{B} T=2.59 \hbar \omega$ in Fig. 3(f).

There are two central reasons for presenting these redox DOS and rate results in the single-particle picture. ${ }^{14,18,31,38,41}$ First, the low temperature trends can be utilized to arrive at a redox based single-particle description of the low temperature Franck-Condon blockade. ${ }^{4}$ Second, the rather low zero bias electron transfer rates with large reorganization energies can be used to argue that Franck-Condon blockade should be observable also at room temperature-where it is essentially two-electrode redox chemistry. ${ }^{19-21,23}$ Both of these aspects will be explored next.

\section{FRANCK-CONDON BLOCKADE AND REDOX CHEMISTRY}

As described in the Introduction, Franck-Condon blockade is essentially Coulomb blockade in the strong electronphonon coupling regime $(\zeta>0) .{ }^{4}$ In either blockade regime, the species under investigation is subjected to current flow between two contacts biased at $V_{B}=\mu_{L}-\mu_{R}$ as shown in Fig. 4-where $\mu_{L}$ and $\mu_{R}$ are the electrochemical potentials of the left and right contacts, respectively. A further insulating gate electrode $V_{G}$ is applied to vary the position of a species' single-particle spectrum relative to that of $\mu_{L}$ and $\mu_{R}$ (see Fig. 4).

Conduction between the left and right contacts is mediated by tunneling to/from the species under investigation. These tunneling events can be described by forward and backward electron transfer rates $k_{f, L}, k_{b, L}, k_{f, R}$, and $k_{b, R}$ as depicted in Fig. 4. The respective electron transfer rates for each contact are arrived at by substituting $\mu_{L}$ or $\mu_{R}$ (as is appropriate) into
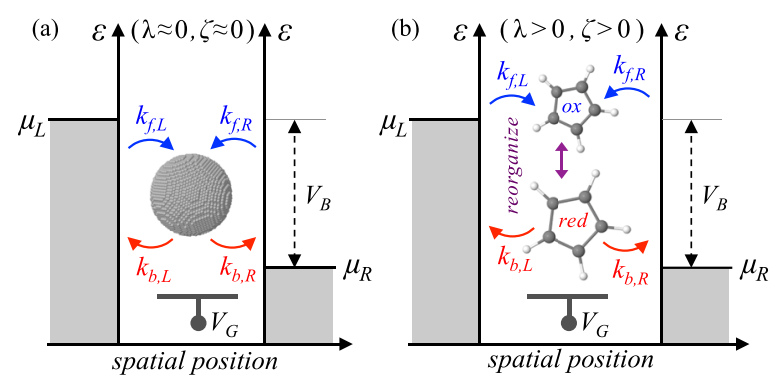

FIG. 4. (a) Coulomb blockade and (b) Franck-Condon blockade from the perspective of electron transfer theory, considering only transitions between oxidized ( $\mathrm{N}-1$ electrons) and reduced ( $\mathrm{N}$ electrons) states. 
Eq. (1), (2), (7), or (8). For a single-level transitioning between $N-1$ (oxidized) and $N$ (reduced) charge states, the overall electron flow is given by

$$
I_{e}=e\left[\left(k_{f, L} P_{o x}-k_{b, L} P_{r e d}\right)-\left(k_{f, R} P_{o x}-k_{b, R} P_{r e d}\right)\right] / 2,
$$

where $P_{o x}=P(N-1)$ and $P_{\text {red }}=P(N)$ are the probabilities of being in the $N-1$ (oxidized) and $N$ (reduced) charge states, respectively. Here we assume that the species undergoing electron transfer fully relaxes thermally prior to undergoing any subsequent electron transfer event (as is typical in the electron transfer literature) ${ }^{31,37,38}$ To solve for the probability terms in Eq. (14), we utilize the master equation approach ${ }^{2}$ in the simplified two-state form

$$
\begin{gathered}
P_{o x}+P_{r e d}=1, \\
\left(k_{f, L}+k_{f, R}\right) P_{o x}=\left(k_{b, L}+k_{b, R}\right) P_{r e d}
\end{gathered}
$$

which dictates a steady-state probability and rate conservation for a system transitioning between $N-1$ (oxidize) and $N$ (reduced) charge states.

In the characterization of blockade phenomena, typically one plots the absolute rate of change in electron/current flow ( $\left|d I_{e} / d V_{B}\right|$, i.e., differential conductance) with respect to the two-electrode applied bias $V_{B}$. This is done at various gate voltages $\left(V_{G}\right)$, as shown in Fig. 5, to provide a direct measure of the two-electrode biases over which species' levels fall resonant between $\mu_{L}$ and $\mu_{R}$. Note, in Fig. 5, it is assumed that the electronic coupling $\left(|M|^{2}\right)$ is the same for both contacts. If such a plot is extended to multiple charge states (beyond $N-1$ and $N$ ), then it takes the form of what is called a "Coulomb blockade diamond." $3,4,10,11,42$

In Figs. 5(a)-5(c), Coulomb blockade plots are provided for transitions between $N-1$ and $N$ electrons in the low electron-phonon coupling regime $(\lambda \approx 0, \zeta \approx 0)$ with electron transfer rates governed by Eqs. (1) and (2). These three plots show the evolution of conductance though a singlelevel $\left(\varepsilon_{d}\right)$ from the low temperature limit $\left[k_{B} T_{\text {low }}=0.0259 \hbar \omega\right.$ in Fig. 5(a)] through to the high temperature limit $\left[k_{B} T_{\text {high }}\right.$ $=2.59 \hbar \omega$ in Fig. 5(c)] and also at an intermediate temperature $\left[k_{B} T_{i n t}=0.664 \hbar \omega\right.$ in Fig. 5(b)]. This conductance regime is representative of what might be observed for a mesoscopic system $^{8}$ [such as that illustrated in Fig. 4(a)]. At low temperatures, conductance is sharply peaked around $\left(\varepsilon_{d}\right)$ as it is gated by $V_{G}$ [see Fig. 5(a)]. However, as the Fermi distribution of both contacts begins to broaden so does the conductance
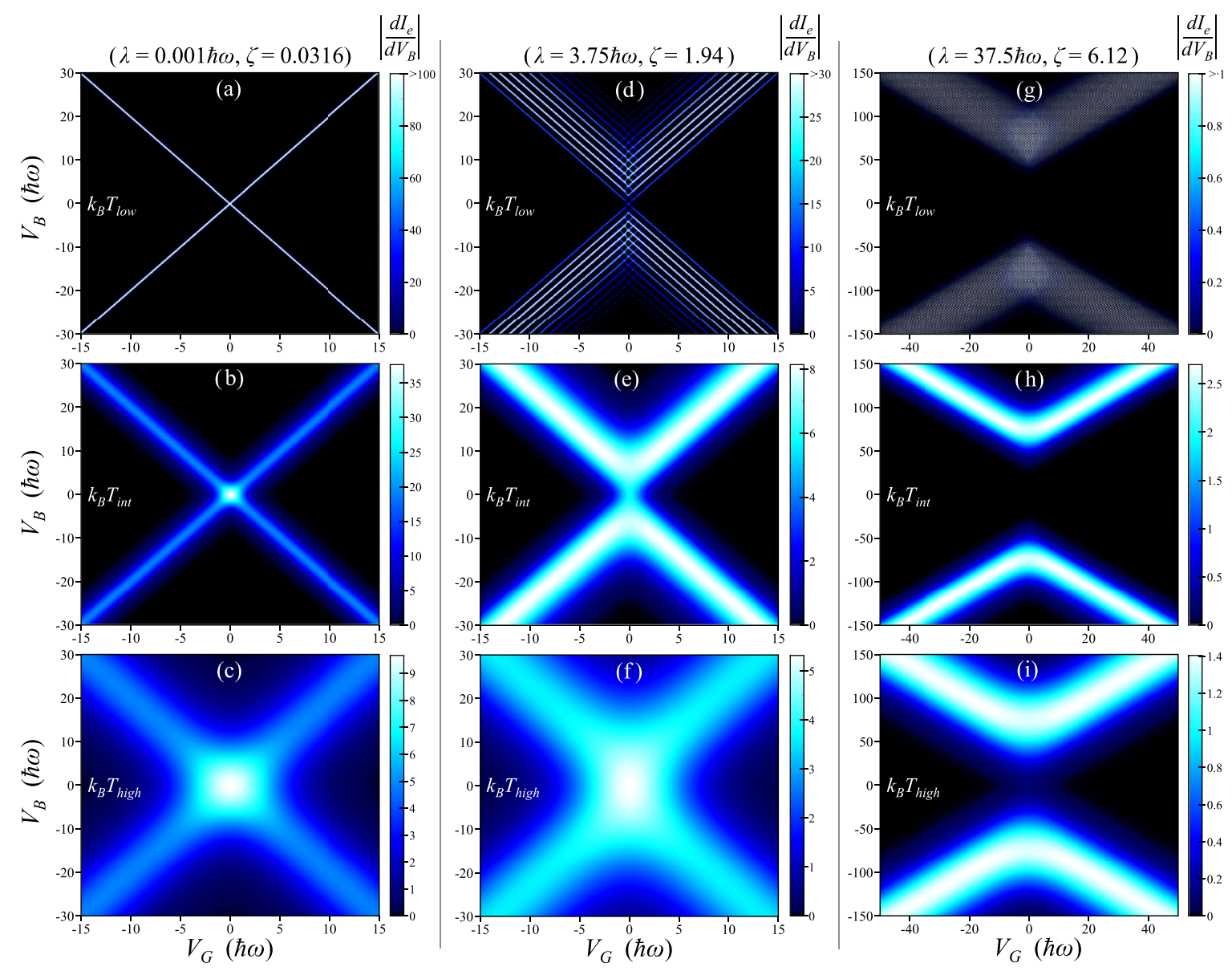

FIG. 5. Blockaded electron transport $\left|d I_{e} / d V_{B}\right|$ differential conductance plots, with the maximum electron current magnitude $\left(\left|I_{e}\right|_{\text {max }}\right)$ normalized to 1 , as a function of electron-phonon coupling and temperature. [(a)-(c)] Coulomb blockade features through a "mesoscopic particle" transitioning between $N-1$ and $N$ electrons with $\zeta=0.0316$ for (a) $k_{B} T_{\text {low }}=0.0259 \hbar \omega$, (b) $k_{B} T_{\text {int }}=0.664 \hbar \omega$, and (c) $k_{B} T_{\text {high }}=2.59 \hbar \omega$. [(d)-(f)] Franck-Condon blockade through a molecule transitioning between $N-1$ and $N$ electrons, oxidation and reduction, with $\zeta=1.94$ for (d) $k_{B} T_{\text {low }}=0.0259 \hbar \omega$, (e) $k_{B} T_{\text {int }}=0.664 \hbar \omega$, and (f) $k_{B} T_{\text {high }}=2.59 \hbar \omega$. [(g)-(i)] Franck-Condon blockade features for a molecule with $\zeta=6.12$ for (g) $k_{B} T_{\text {low }}=0.0259 \hbar \omega$, (h) $k_{B} T_{\text {int }}=0.664 \hbar \omega$, and (i) $k_{B} T_{\text {high }}=2.59 \hbar \omega$. All results are for a simplified single-mode phonon model, assuming symmetric electronic coupling $|M|^{2}$ for each contact. The reorganization energy is related to $\zeta$ via $\lambda=\zeta^{2} \hbar \omega$. 
range, resulting in a gradual smoothening around $T_{\text {int }}$ as shown in Fig. 5(b). Eventually, at high temperatures (e.g., $T_{\text {high }}$ ), the differential conductance becomes quite smeared as shown in Fig. 5(b), without sharp transition biases for conductance through $\varepsilon_{d}$.

These same Fermi smearing trends, with increasing temperature, are present in the strong electron-phonon coupling regime $(\lambda>0, \zeta>0)$ that characterizes Franck-Condon blockade as shown in Figs. 5(d)-5(i). To capture FranckCondon blockade, we still describe current flow via Eqs. (14), (15), and (16)—see also Fig. 4(b). However, the contact electron transfer rates are governed by the more general descriptions given by Eqs. (7) and (8). This provides for the emergence of discrete electron transfer single-particle energy levels at intervals separated by $\hbar \omega$ off resonant from $\varepsilon_{d}$, as was discussed in the context of Eqs. (9) and (10) and presented earlier in Fig. 3.

Let us first consider Franck-Condon blockade in the moderate electron-phonon coupling regime $(\lambda=3.75 \hbar \omega$, $\zeta=1.94$ ) as shown in Figs. 5(d)-5(f). At $T_{\text {low }}$ in Fig. 5(d), we can see a clear suppression of zero bias conductance due to electron-phonon coupling (i.e., molecular reorganization) and a crisscrossed diamond structuring in conduction arising from the quantized distributions of $D_{o x}$ and $D_{\text {red }}$ [see also Fig. 3(a)]. These are the clear features delineated by phonon quantization $(\hbar \omega)$ that are typically associated with Franck-Condon blockade. ${ }^{4,9-11}$ Note that, the spacing between Franck-Condon blockade peaks is actually $2 \hbar \omega$ in Fig. 5(d). This is because an oxidation state and a reduction state (both spaced by $\hbar \omega$ ) must both be accessed within the bias window of $V_{B}$ for the current to increase [see Fig. 4(b)]. However, as the temperature is increased to $T_{\text {int }}$, these quantization features are largely suppressed by Fermi broadening the contacts, as shown in Fig. 3(b). However, a slight suppression in the zero bias conductance remains at $T_{i n t}$ when $\lambda=3.75 \hbar \omega$. Finally, once the temperature is increased to $T_{\text {high }}$, all traces of Franck-Condon blockade are lost when $\lambda=3.75 \hbar \omega$, with the highest conductance now present at zero bias [see Fig. 5(f)]. The features in Fig. 5(f) are not too dissimilar from those in Fig. 5(d), with the broadening of $D_{o x}$ and $D_{\text {red }}$ contributing further to smearing of differential conductance when $\lambda>0$ [see also Fig. 3(b)].

Now, if we increase the reorganization energy of our species to $\lambda=37.5 \hbar \omega$ (corresponding to $\zeta=6.12$ for this single mode toy-model), a markedly different temperature trend is obtained in Figs. 5(g)-5(i). Again, we see the Franck-Condon suppression of low bias conductance at $T_{\text {low }}$ in Fig. $5(\mathrm{~g})$. However, as the temperature is increased to $T_{\text {int }}$ and $T_{\text {high }}$ in Figs. 5(h) and 5(i), respectively, we see that zero bias conductance remains suppressed. Thus, even in the presence of Fermi smearing in the contacts and redox DOS broadening at higher temperatures [see Fig. 3(e)], it should be possible to observe Franck-Condon blockade. This high temperature regime of "Franck-Condon" blockade is characterized primarily by low bias current suppression. ${ }^{4,9,19-21}$ Due to Fermi smearing in the contacts, the high temperature regime lacks the crisscrossed diamond structuring of differential conductance [such as that shown Fig. 5(d)] via transport through successive single-particle energies/levels separated by $\hbar \omega .{ }^{10,11}$
In short, the high temperature Franck-Condon blockade can be viewed as two electrode redox chemistry mediated by tunneling electron transfer. ${ }^{19-21}$ In the same spirit, one can also argue that the low temperature Franck-Condon blockade is, analogously, a form of low temperature redox chemistry. 11,22,39 The very low electron transfer rates that are present at low biases when $\lambda \gg 0$ form the central reason for high temperature current suppression at low biases. As shown in Fig. 3(f), when $\lambda=37.5 \hbar \omega$, we have a low bias electron transfer rate that is a small fraction $\left(<10^{-2}\right)$ of the high bias rate. This is not the case for small reorganization energies [as shown in Fig. 3(c)]. Thus, high temperature Franck-Condon blockade is limited to reactants/species with large reorganization energies.

Experimentally, even greater asymmetries between low bias and high bias redox electron transfer rates have been observed at room temperature [than that given in Fig. 3(f), resulting in Fig. 5(i)]. For example, in the seminal measurements by Chidsey ${ }^{30,32}$ conducted on ferrocene situated atop an alkane monolayer, the room temperature low bias electron transfer rate was observed to be more than $10^{-3}$ times smaller than the high bias electron transfer rate. Thus, it is plausible that ferrocene situated between two tunneling monolayers could exhibit the high temperature Franck-Condon blockade features displayed in Fig. 5(i). Most room temperature tunneling redox experiments are performed through ionic "gating" via a supporting electrolyte, ${ }^{30,38,43-45}$ whereas low temperature Franck-Condon blockade experiments have typically made use of solid-state electrostatic gating. ${ }^{10,11}$ The ionic gating of electrical devices has attracted great interest in the solid-state device physics community; ${ }^{46,47}$ thus, there is no reason why similar methods might not be applied to observe room temperature Franck-Condon blockade.

It should be noted, however, that the reorganization energies typically observed in room temperature redox experiments follow from the summation of reorganization energies across many phonon modes $\left(\lambda=\sum_{n} \lambda_{n}\right.$, where $n$ is the phonon mode index). ${ }^{34,35,37}$ Thus, the low temperature current suppression trends displayed in Fig. 5(g) are unlikely to be observed upon cooling down a reactant such as ferrocene. Rather, one may expect that the lowest energy mode would begin to dominate electron transfer at low biases, and features more in agreement with Fig. 5(d) would be observed at low temperatures (in molecular systems such as ferrocene). ${ }^{10,11,22}$ This discrepancy exists in our theoretical model because we have used a single-mode toy model (to simplify the discussion and focus on the essential physics). A multi-mode model is left for future work.

\section{CONCLUSION}

In this work, we have explored an electron transport oriented connection between redox chemistry and FranckCondon blockade. This was accomplished by revisiting singleparticle quantum mechanical expressions of electron transfer between a molecule/reactant and a metal contact. ${ }^{4}$ In our analysis, the molecular/reactant nuclear degrees of freedom were quantized in a single phonon mode description, such that the redox DOS becomes quantized via Franck-Condon coupling 
terms in intervals of $\hbar \omega$-Planck's constant multiplied by the mode frequency. In the high temperature limit, it was shown that the quantum mechanical redox DOS is well approximated by the semi-classical redox DOS expressions developed by Gerischer and Hopfield. ${ }^{14-18,31}$ Correspondingly, the quantum mechanical and semi-classical electron transfer rate expressions were shown to agree well in the high temperature limit. In the low temperature limit, the quantized redox DOS gives rise to discrete steps in the electron transfer rate separated by $\hbar \omega$ and correspondingly to characteristic Franck-Condon blockade diamond features in a gated two electrode system. At small reorganization energies $(\lambda)$, these Franck-Condon blockade features were shown to wash out as the temperature is increased, due to Fermi smearing in the contacts. Subsequently, it was shown that in the large reorganization energy limit $\lambda \gg 0$, the rate of electron transfer at low biases may be significantly suppressed such that Franck-Condon blockade might be observed even in the high temperature regime. Extrapolating directly from the seminal electron transfer work by Chidsey ${ }^{30}$ it is argued that this high temperature FranckCondon blockade physics may be observed in gated two electrode system consisting of ferrocene sandwiched between two suitably thick monolayers-or similar such molecular configurations. ${ }^{19-22}$

\section{SUPPLEMENTARY MATERIAL}

See supplementary material for extended derivations pertaining to rate expressions.

\section{ACKNOWLEDGMENTS}

Financial support from NSERC of Canada and FQRNT of Québec is gratefully acknowledged.

${ }^{1}$ M. Thoss and F. Evers, J. Chem. Phys. 148, 030901 (2018).

${ }^{2}$ S. Datta, Quantum Transport: Atom to Transistor (Cambridge University Press, 2005).

${ }^{3}$ B. Muralidharan, A. W. Ghosh, and S. Datta, Phys. Rev. B 73, 155410 (2006).

${ }^{4}$ J. Koch, F. von Oppen, and A. V. Andreev, Phys. Rev. B 74, 205438 (2006).

${ }^{5}$ X. H. Qiu, G. V. Nazin, and W. Ho, Phys. Rev. Lett. 92, 206102 (2004).

${ }^{6}$ S. Chen, R. S. Ingram, M. J. Hostetler, J. J. Pietron, R. W. Murray, T. G. Schaaff, J. T. Khoury, M. M. Alvarez, and R. L. Whetten, Science 280, 2098 (1998).

${ }^{7}$ F.-R. F. Fan and A. J. Bard, Science 277, 1791 (1997).

${ }^{8}$ J. Kane, J. Ong, and R. F. Saraf, J. Mater. Chem. 21, 16846 (2011).

${ }^{9}$ J. Koch and F. von Oppen, Phys. Rev. Lett. 94, 206804 (2005).

${ }^{10}$ E. Burzurí, Y. Yamamoto, M. Warnock, X. Zhong, K. Park, A. Cornia, and H. S. J. van der Zant, Nano Lett. 14, 3191 (2014).
${ }^{11}$ C. S. Lau, H. Sadeghi, G. Rogers, S. Sangtarash, P. Dallas, K. Porfyrakis, J. Warner, C. J. Lambert, G. A. D. Briggs, and J. A. Mol, Nano Lett. 16, 170 (2015).

${ }^{12}$ J. Franck and E. G. Dymond, Trans. Faraday Soc. 21, 536 (1926).

${ }^{13}$ E. Condon, Phys. Rev. 28, 1182 (1926).

${ }^{14}$ J. J. Hopfield, Proc. Natl. Acad. Sci. U. S. A. 71, 3640 (1974).

${ }^{15}$ H. Gerischer, Z. Phys. Chem. 26, 223 (1960).

${ }^{16}$ H. Gerischer, Z. Phys. Chem. 26, 325 (1960).

${ }^{17}$ H. Gerischer, Z. Phys. Chem. 27, 48 (1961).

${ }^{18}$ K. H. Bevan, J. Chem. Phys. 146, 134106 (2017).

${ }^{19}$ I. G. Medvedev, J. Electroanal. Chem. 660, 285 (2011).

${ }^{20}$ I. G. Medvedev, J. Chem. Phys. 141, 124706 (2014).

${ }^{21}$ J. Zhang, A. M. Kuznetsov, I. G. Medvedev, Q. Chi, T. Albrecht, P. S. Jensen, and J. Ulstrup, Chem. Rev. 108, 2737 (2008).

${ }^{22}$ R. Arielly, M. Vadai, D. Kardash, G. Noy, and Y. Selzer, J. Am. Chem. Soc. 136, 2674 (2014).

${ }^{23}$ A. M. Kuznetsov and I. G. Medvedev, Phys. Rev. B 78, 153403 (2008)

${ }^{24}$ A. M. Kuznetsov and J. Ulstrup, J. Phys. Chem. A 104, 11531 (2000).

${ }^{25}$ W. Schmickler, Surf. Sci. 295, 43 (1993).

${ }^{26}$ A. M. Kuznetsov, Charge Transfer in Physics, Chemistry, and Biology: Physical Mechanisms of Elementary Processes and an Introduction to the Theory (Gordon and Breach Publishers, 1995).

${ }^{27}$ V. G. Levich, "Kinetics of reactions with charge transfer," in Physical Chemistry: An Advanced Treatise, Vol. Xb (Academic Press, New York, 1970).

${ }^{28}$ K. H. Bevan, D. Kienle, H. Guo, and S. Datta, Phys. Rev. B 78, 035303 (2008).

${ }^{29} \mathrm{~N}$. Abraham, "Electronic tunnel factors in molecular electron transfer and molecular conduction," in Encyclopedia of Electrochemistry (American Cancer Society, 2007).

${ }^{30}$ C. E. D. Chidsey, Science 251, 919 (1991).

${ }^{31} \mathrm{~K}$. H. Bevan, M. S. Hossain, A. Iqbal, and Z. Wang, J. Phys. Chem. C 120, 179 (2016).

${ }^{32}$ M. S. Hossain and K. H. Bevan, J. Phys. Chem. C 120, 188 (2016).

${ }^{33} \mathrm{The}$ symbol $\lambda$ is reserved for denoting reorganization energies, as is common in the chemistry literature.

${ }^{34}$ W. Schmickler, M. A. Rampi, E. Tran, and G. M. Whitesides, Faraday Discuss. 125, 171 (2004).

${ }^{35}$ W. Schmickler, Chem. Phys. 289, 349 (2003).

${ }^{36} \mathrm{~S}$. Datta, Electronic Transport in Mesoscopic Systems (Cambridge University Press, 1995).

${ }^{37}$ W. Schmickler, Interfacial Electrochemistry, 1st ed. (Oxford University Press, 1996).

${ }^{38}$ M. S. Hossain, B. Muralidharan, and K. H. Bevan, J. Phys. Chem. C 121, 18288 (2017).

${ }^{39}$ A. Roy-Gobeil, Y. Miyahara, K. H. Bevan, and P. Grutter, "Fully quantum mechanical electron transfer observed in a single redox molecule at a metal interface" (unpublished).

${ }^{40}$ S. De Franceschi, S. Sasaki, J. M. Elzerman, W. G. van der Wiel, S. Tarucha, and L. P. Kouwenhoven, Phys. Rev. Lett. 86, 878 (2001).

${ }^{41}$ A. Migliore, P. Schiff, and A. Nitzan, Phys. Chem. Chem. Phys. 14, 13746 (2012).

${ }^{42}$ J. S. Seldenthuis, H. S. J. van der Zant, M. A. Ratner, and J. M. Thijssen, ACS Nano 7, 1445 (2008).

${ }^{43}$ M. S. Hossain, A. Iqbal, and K. H. Bevan, Anal. Chem. 88, 9062 (2016).

${ }^{44}$ M. J. Honeychurch, Langmuir 15, 5158 (1999).

${ }^{45}$ E. Tran, M. A. Rampi, and G. M. Whitesides, Angew. Chem., Int. Ed. 43, 3835 (2004).

${ }^{46}$ A. Herklotz et al., Nano Lett. 17, 1665 (2017).

${ }^{47}$ T. Fujimotoa and K. Awaga, Phys. Chem. Chem. Phys. 15, 8983 (2013). 